\title{
Crystal structure of a monoclonal antibody directed against an antigenic determinant common to Ogawa and Inaba serotypes of Vibrio cholerae 01
}

Firoz Ahmed ${ }^{1,2}$, Gwénaëlle André-Leroux ${ }^{1}$, Ahmed Haouz ${ }^{1}$, Alain Boutonnier ${ }^{1}$, Muriel Delepierre $^{1}$, Firdausi Qadri ${ }^{2}$, Farida Nato ${ }^{1}$, Jean-Michel Fournier ${ }^{1}$, Pedro M. Alzari ${ }^{1}$ *

${ }^{1}$ Institut Pasteur \& CNRS URA 2185, 25/28 rue du Dr. Roux, Paris, France; ${ }^{2}$ International Centre for Diarrhoeal Disease Research, Bangladesh (ICDDR,B), Dhaka, Bangladesh.

*Author to whom correspondence should be addressed:

Pedro M. Alzari

Unité de Biochimie structurale, Institut Pasteur

25 rue du Docteur Roux, 75724 Paris, France

Phone: (33) 1456886 07, Fax: (33) 145688604

Email: alzari@pasteur.fr

\section{Keywords}

Vibrio cholerae, LPS, cholera, anti-LPS antibody, crystal structure

Running title: Crystal structure of an anti-cholera antibody 


\section{Introduction}

Cholera remains a major public health problem. Although the improvement of hygiene is an efficient mechanism for the long-term control of the disease, this is still a distant goal in many developing countries where an efficient, simple vaccine could be of great help. The lipopolisaccharide (LPS) of Vibrio cholerae serogroup O1, which is responsible for most cholera outbreaks, is the most consistently described antigen associated with protection of humans from cholera ${ }^{1}$. The O1-LPS is composed of a hydrophobic lipid portion, lipid A, and a hydrophilic polar region consisting of the core polysaccharide (core-PS) and the O-antigen polysaccharide (O-PS), a long regular chain of $\alpha(1,2)$-linked D-perosamine residues ${ }^{2}$. The O1-LPS contains at least two major antigenic determinants. Monoclonal antibodies specific for the Ogawa serotype recognize the upstream (non-reducing) terminal O-PS perosamine residue as the primary antigenic determinant. The crystal structure of the Fab fragment of a monoclonal antibody directed against this antigenic determinant has been determined in complex with synthetic fragments of the Ogawa O-specific polysaccharide ${ }^{3}$, providing useful hints to understand the structural basis of antigen specificity ${ }^{4}$.

A second determinant is common to the two major V. cholerae O1 serotypes (Ogawa and Inaba), but it is unclear whether the core-PS and/or the O-PS are involved in this antigenic determinant. Using a series of conjugates of the polysaccharide moiety of the lipopolysaccharide of $V$. cholerae $O 1$, serotype Inaba, we produced monoclonal antibodies that recognize this common epitope and used them to develop a rapid diagnostic test for cholera ${ }^{5-7}$. We report here the crystal structure of the Fab fragment from one of these antibodies, IgG1 F22-30, and 
determined its crystal structure at $2.5 \AA$ resolution. The structure revealed the presence of a putative binding pocket in the recombination site, suggesting that the antibody may specifically bind either a terminal sugar from the core-PS or a perosamine side-chain from the O-PS. Preliminary molecular mechanics calculations and NMR studies suggest that perosamine residues could indeed play a role in molecular recognition.

\section{Materials and Methods}

Monoclonal Mouse Ab F-22-30 (IgG1, $\kappa)$ was purified from the ascitic fluid by protein-Gagarose affinity chromatography. The molecule was eluted with glycine- $\mathrm{HCl}(100 \mathrm{mM}, \mathrm{pH} 2.5)$ and equilibrated against 2.0 M Tris-base at $\mathrm{pH}$ 9.0. The Fab fragment was produced by papain digestion with an enzyme:substrate molar ratio of 1:30 in $0.1 \mathrm{M}$ phosphate buffer, $\mathrm{pH} 7.2,2.5$ $\mathrm{mM}$ EDTA, $10 \mathrm{mM}$ L-cysteine at $37^{\circ} \mathrm{C}$. The Fab fragment was separated by ion-exchange

chromatography and further purified by gel filtration on a Superdex ${ }^{\mathrm{TM}}$ G75 column (Amersham Biosciences). As isoelectric focusing revealed the presence of heterogeneity, homogeneous Fab peaks were separated by an additional step of ion-exchange chromatography in a Mono-Q DEAE column. The purified Fab fragment was concentrated to $10 \mathrm{mg} / \mathrm{ml}$ in $40 \mathrm{mM}$ Tris- $\mathrm{HCl}, \mathrm{pH} 7.6$, for crystallization trials.

Crystals were obtained using the hanging drop method by mixing $1.5 \mu \mathrm{l}$ of protein solution and $1.5 \mu \mathrm{l}$ of the reservoir solution containing $18 \%(\mathrm{~V} / \mathrm{V})$ of PEG 4000, 0.1M Na Citrate, $0.2 \mathrm{M}$ magnesium sulfate, $\mathrm{pH}$ 5.0. These crystals grew to a maximum dimension of $0.1 \mathrm{~mm} \mathrm{x} 0.1 \mathrm{~mm} \mathrm{x}$ $0.4 \mathrm{~mm}$ within two weeks at $18^{\circ} \mathrm{C}$. Crystals are triclinic, space group P1, with cell dimensions 
$a=75.06 \AA, b=75.22 \AA, c=98.41 \AA, \alpha=111.1^{\circ}, \beta=90.4^{\circ}$ and $\gamma=106.8^{\circ}$. X-ray diffraction data at 2.5 $\AA$ resolution were collected on beamline ID29 at the ESRF (Grenoble) from a frozen crystal at $100 \mathrm{~K}$ in mother liquor $+20 \%(\mathrm{v} / \mathrm{v})$ glycerol. Data reduction was carried out with the programs MOSFLM and SCALA from the CCP4 software package ${ }^{8}$. Four copies of the Fab fragment were positioned in the asymmetric unit by molecular replacement methods with the program AMoRe ${ }^{9}$ using the structures of different Fab fragments from the PDB as search probes. Refinement was carried out with REFMAC ${ }^{10}$ using a TLS model (each variable and constant domain of the Fab fragments was considered as a separate rigid body) alternated with rounds of validation and rebuilding with the program $\operatorname{Coot}^{11}$. The final atomic models include the complete light $(\mathrm{L})$ and heavy $(\mathrm{H})$ chains, except for the C-terminal disulfide bridge linking Cys219L and Cys $220 \mathrm{H}$ and the protein loop H132-H140 from the constant region of the heavy chain, which are not visible in the electron density maps. No water molecules were introduced in the model. The experimental structure factors and the refined coordinates for the final model (Table 1) have been deposited in the PDB with accession code 2UYL.

Molecular modelling calculations were carried out using the InsightII, Biopolymer and Discover modules of the Accelrys $\odot$ package (Accelrys Inc., San Diego, CA, USA). All energy optimizations were done with the CFF91 force field as described ${ }^{12}$. Four distinct terminal sugar residues ( $\alpha$-glucose, glucosamine, $\beta$-fructose and $\alpha$-heptose) from the core-PS, in each case linked to a glucose residue, and a D-perosamine (4-amino-4,6-dideoxy- $\alpha$-D-mannopyranosyl) acylated on its amino group with 3-deoxy-L-glycero-tetronic acid were manually docked in the putative monosaccharide-binding pocket of the antibody recombination site. Different initial positions for each complex were then subjected to energy minimization, with the main-chain atoms of the Fab molecule constrained to their crystallographic positions. The total energies of 
the optimal position for each complex are given in Table 2. The graphic figures were created with PyMOL (DeLano Scientific).

${ }^{1} \mathrm{H}$ NMR experiments were recorded at $308 \mathrm{~K}$ on Varian Unity Inova spectrometers operating at ${ }^{1} \mathrm{H}$ frequencies of $500 \mathrm{MHz} .{ }^{1} \mathrm{H}$ chemical shifts were referred to external DSS. Three millimeters Shigemi tubes were used for all samples. For the preparation of a NMR sample, 0.6 mg of LPS form $V$. cholera Inaba were dissolved in $100 \mu \mathrm{D}_{2} \mathrm{O}$ leading to a concentration of about $0.9 \mathrm{mM}$. IgG1 antibodies were prepared from a stock solution at $4 \mathrm{mg} / \mathrm{ml}$. STD experiments were recorded at $308 \mathrm{~K}$. For all saturation transfer difference experiments (STDNMR), selective saturation of antibody resonances were performed at $0.28 \mathrm{ppm}, 0.5 \mathrm{ppm}$ and 7 ppm (20 ppm for reference spectra) using a series of 40 Gaussian shaped pulses $(50 \mathrm{~ms}, 10 \mathrm{~ms}$ delay between pulses, excitation width approximately $50 \mathrm{~Hz}$ ). Substraction of saturated spectra from reference spectra was performed by phase cycling ${ }^{13}$.

\section{Results and Discussion}

The crystal structure of the Fab fragment from monoclonal antibody F-22-30 directed against $V$. cholerae O1-LPS has been determined at $2.5 \AA$ resolution (Figure 1a) and refined to a final crystallographic R-factor (R-free) of 0.211 (0.257) (Table 1). The four independent molecules in the unit cell are very similar to each other, with rmsd values of $0.1-0.2 \AA$. The model displays a good stereochemistry, with a single protein residue (Ser85H) falling out of the allowed regions in the Ramachandran plot ${ }^{11}$. However, this residue has the same unfavorable 
conformation and has a well-defined electron density in all four independent Fab molecules, suggesting that this is a genuine feature of the structure.

The antigen-binding site displays a rather shallow surface, with two bulges corresponding to the tips of the $\mathrm{L} 1$ and $\mathrm{H} 2$ hypervariable loops and a small deep cavity primarily defined by residues from the L1, L3 and H3 hypervariable loops (Figure 1b). This putative binding pocket, delimited by the aromatic face of Tyr37L, the main-chain residues of both $\mathrm{L} 3$ and $\mathrm{H} 3$, and the side chains of salt-bridged residues Arg101L and Glu99H, appears to be occupied by an unidentified molecule in the crystal (Figure 1c). The size and shape of the pocket suggests that it could accommodate a single (terminal) monosaccharide from the O1-LPS. The core-PS contains four such putative epitopes, namely $\alpha$-glucose, glucosamine, $\alpha$-heptose and $\beta$-fructose ${ }^{2,14}$. Another possible ligand for this pocket could be the side-chain (3-deoxy-L-glycero-tetronic acid) that acylates the amino group of perosaminyl residues from the O-PS.

Preliminary molecular modeling calculations were carried out for these five putative ligands, as described in Materials and Methods. The results suggest that all five molecules could occupy the observed pocket, in some cases with the sugar ring making stacking interactions with Tyr37L. In all predicted models, a few protein residues (His31L, Arg101L, His50H, Glu99H and Thr101H) could be involved in protein-ligand hydrogen bonding interactions. As shown in Table 2 , the best binding affinity is predicted for the D-perosamine side-chain. This ligand is completely anchored in the binding pocket, where it could interact with the aromatic face of Tyr37L and form several hydrogen bonding interactions with residues from the light and heavy chain of the antibody (Figure 2). Interestingly, the perosamine side-chain is also the only compound predicted 
to form a hydrogen bond with Glu39L, which is located at the bottom of the cavity (Figure 1c) and cannot interact with the other ligands.

NMR experiments provide some additional support for the model predictions. As shown in Figure 3, perosamine resonances are clearly observed in the STD-NMR spectra obtained with onresonance irradiations of antibody resonances, either in the methyl proton region (.5 ppm, Figure $3 b)$ or in the aromatic proton region $(7 \mathrm{ppm}$, Figure $3 \mathrm{c})$. It is interesting to note that protons from both the ring and the side-chain $(2.05 \mathrm{ppm}$ and 1,87 ppm), although much weaker, are observed, suggesting that the whole perosamine is in contact with the antibody, in agreement with the docking results. The unfavorable ratio of perosamine units to core units precludes a more detailed analysis of the observed peaks in the STD-NMR spectra and cannot exclude interaction with the core residues.

In conclusion, we report here the first crystal structure of a monoclonal antibody that recognizes a common epitope present in both Ogawa and Inaba serotypes from Vibrio cholerae O1. Modeling calculations and preliminary NMR results suggest that the binding pocket at the center of the antibody recombination site could bind the side-chain of a perosaminyl residue from the O-PS. This would be in agreement with the higher accessibility to antibodies of the O-PS homopolymer compared with the core-PS, and with the observation that antibodies specific for polysaccharides (such as those found in the core-PS) usually display a shallow antigen-binding site $^{15,16}$. If the suggested model holds true, it would imply a lateral recognition of the O-PS antigen, in contrast to antibodies specific for the Ogawa serotype that recognize the terminal, 2O-methylated, perosamine residue ${ }^{3}$. However, further studies of antigen-antibody interactions are clearly required to define the actual epitope since the size and topology of the antibody 
surface would impose structural constraints on the LPS and must necessarily interact with other perosaminyl residues from the homopolymer and/or additional sugar moieties from the core-PS. 


\section{Figure legends}

Figure 1. Crystal structure of Fab F-22-30. A. Final electron density map (contoured at 1.2 $\sigma)$ of a segment from the H3 hypervariable loop (Ser105H-Trp109H). B. Molecular surface of the antibody recombination site. The positions of some hypervariable loops are indicated. Note a deep pocket delimited by the L1, L3 and H3 hypervariable loops. C. A difference Fourier (Fo-Fc) map contoured at $3 \sigma$ suggests that an unidentified molecule binds to this pocket in the crystal.

Figure 2. Putative model of 3-deoxy-L-glycero-tetronic acid docked into the antigenbinding site. Amino acid residues that could participate in protein-ligand H-bonds are indicated. The direction of the O-PS homopolymer would be roughly perpendicular to the plane of the figure.

Figure 3. 1D STD-NMR of Cholera Inaba LPS to IgG at a 50 to $1 \mathrm{LPS}$ to IgG ratio binding site. (a) 1D reference spectrum acquired with a saturation pulse applied at 20 ppm (32 scans; phosphate buffer $50 \mathrm{mM}$; $\mathrm{pH} 6.5 ; 35^{\circ} \mathrm{C} ; 500 \mathrm{MHz}$ ), (b) 1D STD spectrum with on-resonance irradiation of $\mathrm{IgG}$ protons at $0.5 \mathrm{ppm}$ and off-resonance irradiation at $20 \mathrm{ppm}(8192$ scans; phosphate buffer $50 \mathrm{mM}$; pH 6.5; $35{ }^{\circ} \mathrm{C} ; 500 \mathrm{MHz}$ ) (c) 1D STD spectrum with on-resonance irradiation of $\mathrm{IgG}$ protons at $7 \mathrm{ppm}$ and off-resonance irradiation at $20 \mathrm{ppm}(12000$ scans; phosphate buffer $50 \mathrm{mM}$; pH $6.5 ; 35^{\circ} \mathrm{C} ; 500 \mathrm{MHz}$ ). 


\section{References}

1. Provenzano D, Kovac P, Wade WF. The ABCs (antibody, B cells and carbohydrate epitopes) of cholera immunity: Considerations for an improved vaccine. Microbiol Immunol 2006;50:899-927.

2. Chatterjee SN, Chaudhuri K. Lipopolysaccharides of Vibrio cholerae. I. Physical and chemical characterization. Biochim Biophys Acta 2003;1639:65-79.

3. Villeneuve S, Souchon H, Riottot MM, Mazie JC, Lei P, Glaudemans CP, Kovac P, Fournier JM, Alzari PM. Crystal structure of an anti-carbohydrate antibody directed against Vibrio cholerae $\mathrm{O} 1$ in complex with antigen: molecular basis for serotype specificity. Proc Natl Acad Sci USA 2000;97:8433-8438.

4. Chernyak A, Kondo S, Wade TK, Meeks MD, Alzari PM, Fournier JM, Taylor RK, Kovac P, Wade WF. Induction of protective immunity by synthetic Vibrio cholerae hexasaccharide derived from $V$. cholerae O1 Ogawa lipopolysaccharide bound to a protein carrier. J Infect Dis 2002;185:950-962.

5. Boutonnier A, Villeneuve S, Nato F, Dassy B, Fournier JM. Preparation, immunogenicity, and protective efficacy, in a murine model, of a conjugate vaccine composed of the polysaccharide moiety of the lipopolysaccharide of Vibrio cholerae O139 bound to tetanus toxoid. Infect Immun 2001;69:3488-3493.

6. Bhuiyan NA, Qadri F, Faruque AS, Malek MA, Salam MA, Nato F, Fournier JM, Chanteau S, Sack DA, Balakrish Nair G. Use of dipsticks for rapid diagnosis of cholera caused by Vibrio cholerae $\mathrm{O} 1$ and $\mathrm{O} 139$ from rectal swabs. J Clin Microbiol 2003;41:3939-3941. 
7. Nato F, Boutonnier A, Rajerison M, Grosjean P, Dartevelle S, Guenole A, Bhuiyan NA, Sack DA, Nair GB, Fournier JM, Chanteau S. One-step immunochromatographic dipstick tests for rapid detection of Vibrio cholerae $\mathrm{O} 1$ and $\mathrm{O} 139$ in stool samples. Clin Diagn Lab Immunol 2003;10:476-478.

8. CCP4. The CCP4 suite: programs for protein crystallography. Acta Crystallogr D Biol Crystallogr 1994;50:760-763.

9. Navaza J. AMoRe: an automated package for molecular replacement. Acta Crystallogr A 1994;50:157-163.

10. Murshudov GN, Vagin AA, Dodson EJ. Refinement of macromolecular structures by the maximum-likelihood method. Acta Crystallogr D Biol Crystallogr 1997;53:240-255.

11. Emsley P, Cowtan K. Coot: model-building tools for molecular graphics. Acta Crystallogr 2004;60:2126-2132.

12. Andre-Leroux G, Tessier D, Bonnin E. Action pattern of Fusarium moniliforme endopolygalacturonase towards pectin fragments: Comprehension and prediction. Biochim Biophys Acta 2005;1749:53-64.

13. Mayer M, Meyer B. Characterization of ligand binding by saturation transfer difference NMR spectroscopy. Angew Chem Int Ed 1999;38:1784-1788.

14. Vinogradov EV, Bock K, Holst O, Brade H. The structure of the lipid A-core region of the lipopolysaccharides from Vibrio cholerae O1 smooth strain 569B (Inaba) and rough mutant strain 95R (Ogawa). Eur J Biochem 1995;233:152-158.

15. Nguyen HP, Seto NOL, MacKenzie CR, Brade L, Kosma P, Brade H, Evans SV. Germline antibody recognition of distinct carbohydrate epitopes. Nature Struct Biol 2003;10:1019-1025. 
16. van Roon AM, Pannu NS, de Vrind JPM, van der Marel GA, van Boom JH, Hokke CH, Deelder AM, Abrahams JP. Structure of an anti-Lewis X Fab fragment in complex with its Lewis X antigen. Structure 2004;12:1227-1236. 
Table 1. Data collection and refinement statistics.

\begin{tabular}{|c|c|}
\hline Data collection & \\
\hline Data resolution $(\AA)^{*}$ & \\
\hline & $(2.64-2.5)$ \\
\hline Measured reflections ${ }^{*}$ & $127748(18592)$ \\
\hline Unique reflections ${ }^{*}$ & $64354(9355)$ \\
\hline Completeness (\%) ${ }^{*}$ & $97.5(97.1)$ \\
\hline $\mathrm{R}_{\mathrm{sym}}(\%)^{* \dagger}$ & $0.046(0.226)$ \\
\hline$\langle\mathrm{I} / \sigma\rangle^{*}$ & $17.6(5.7)$ \\
\hline Refinement & \\
\hline $\mathrm{R}_{\text {cryst }}{ }^{\dagger}\left[\mathrm{N}^{\circ}\right.$ refs $]$ & $0.211[57803]$ \\
\hline $\mathrm{R}_{\text {free }}{ }^{\dagger}\left[\mathrm{N}^{\circ}\right.$ refs $]$ & $0.256[3275]$ \\
\hline Rms bonds $(\AA)$ & 0.021 \\
\hline Rms angles (degrees) & 1.978 \\
\hline Protein atoms & 13125 \\
\hline Overall B factor $\left(\AA^{2}\right)$ & 23.4 \\
\hline
\end{tabular}

* Values in parentheses apply to the high resolution shell.

$\dagger R_{s y m}=\sum_{h k l} \sum_{i}\left|I_{(}(h k l)-\langle I(h k l)\rangle\right| / \sum_{h k l} \sum_{i} I_{(}(h k l)$

$\$$ Anomalous phasing power $=<[F h($ calc $) V$ phaseintegrated lack of closur $\phi$

括 $R=\sum_{h k l}|F(h) o b s-F(h) c a l c| / \sum_{h k l}|F(h) o b s|$. Rcryst and Rfree were calculated from the working and test reflection sets, respectively. 
Table 2. Total energies of Fab-ligand complexes (referred to that of the Fab-perosamine complex).

Ligand

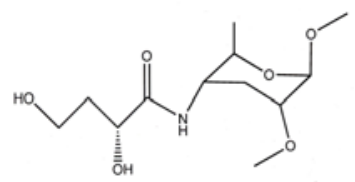

D-Perosamine side-chain

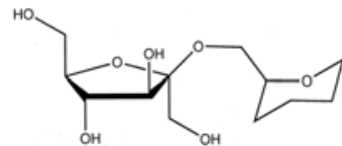

$\beta$-Fructose

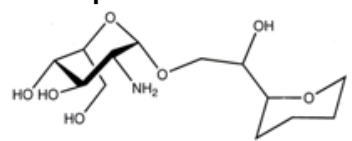

$\alpha$-Glucosamine

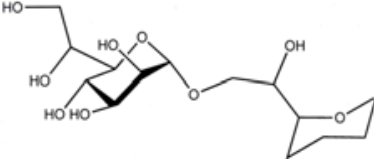

$\alpha$-Heptose

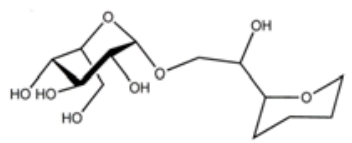

$\alpha$-Glucose

\section{Total energy of the Contribution of} complex (kcal/mol) light / heavy chains

(\%)

$51 / 49$

11.2

37 / 63

23.0

$27 / 73$

25.0

63.4
$53 / 47$

$36 / 64$ 


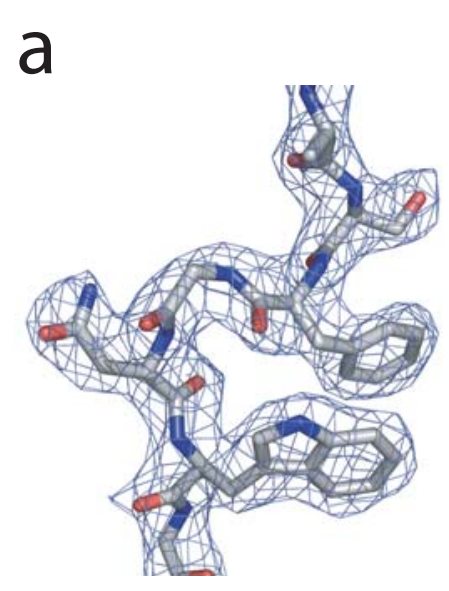

b

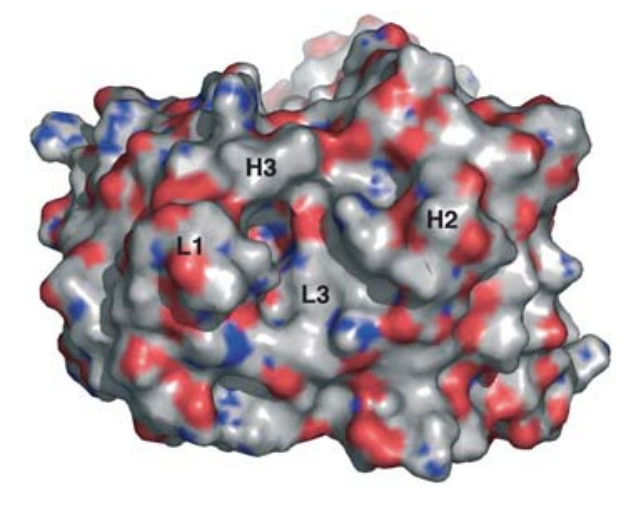

C

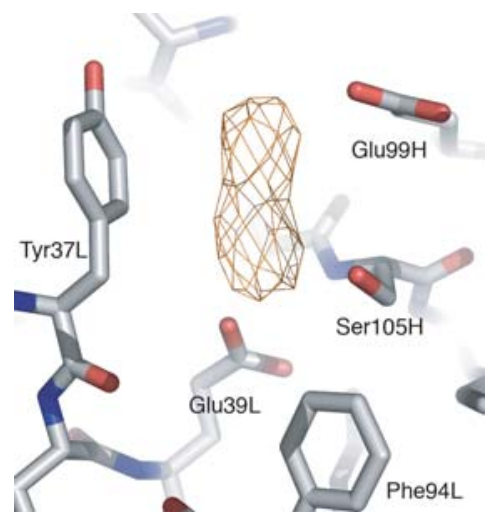

Figure 1 


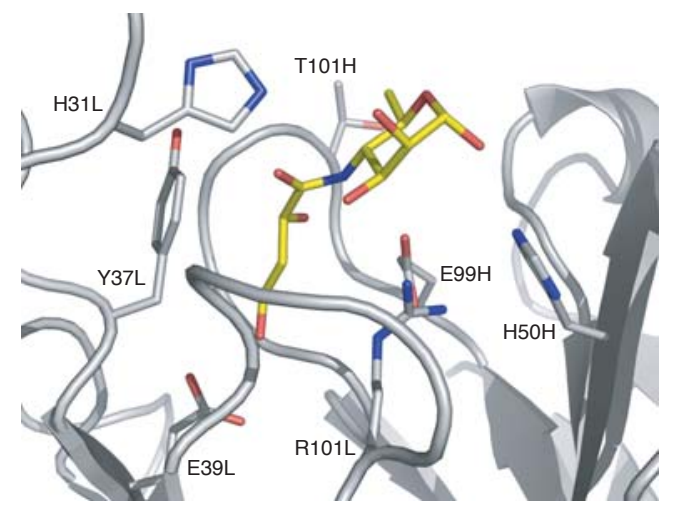

Figure 2 
C

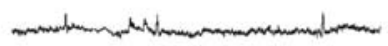

b
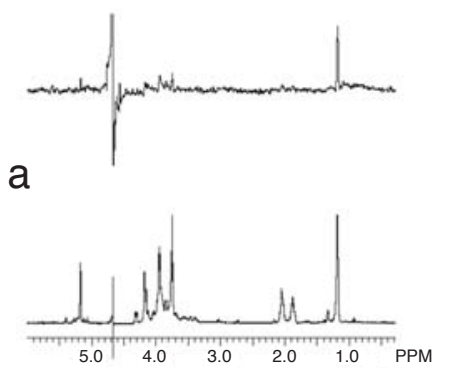

Figure 3 